\title{
Synthesis, Characterization and Thermogravimetric Analysis of 2,2'-Biphenol Based Terpolymer Resins
}

\author{
PRIYANKA U. BELSARE and ANIL B. ZADE* \\ *Professor of Chemistry, Department of Chemistry, Laxminarayan Institute of Technology, \\ Rashtrasant Tukdoji Maharaj, Nagpur University, Nagpur-440 010, India \\ ab_zade18@yahoo.com
}

Received 14 December 2012 / Accepted 13 January 2013

\begin{abstract}
The novel terpolymer resins (2,2'-BPTEPAF- I, II, III) have been synthesized by condensation polymerization of 2,2'-biphenol (2,2'-BP) and tetraethylenepentamine (TEPA) with formaldehyde $(\mathrm{F})$ in presence of $2 \mathrm{M} \mathrm{NaOH}$ as a catalyst using varying molar ratios of reacting monomers. The composition of resins has been determined by elemental analysis. Spectral studies such as UV-Visible, FT-IR and ${ }^{1} \mathrm{H}$ NMR spectra have been used to confine the most probable structures of newly synthesized terpolymer resins. Non-isothermal thermogravimetric analysis has been carried out to determine their mode of decomposition and relative thermal stability. Kinetic parameters such as energy of activation $\left(\mathrm{E}_{\mathrm{a}}\right)$, order of reaction (n) and frequency factor $(\mathrm{z})$ has been calculated by applying Friedman, Chang and Sharp-Wentworth methods. The computed results are compared and elaborated.
\end{abstract}

Keywords: Synthesis, Characterization, Thermogravimetry, Kinetic parameters

\section{Introduction}

Reactions of three component polymeric system by applying suitable method of synthesis have been the subject of interest for many investigators in order to study its high performance utility. In the present study, terpolymer comprises of three components, in which formaldehyde acts as a connecting link between two reacting monomers.

The terpolymer resins showing versatile applications and properties, attracted the attention of scientist and introduced the recent innovations in the polymer chemistry. They are found to be amorphous, crystalline or resinous in nature. The progress in this field has been extremely rapid as they are generally useful in high dielectric constant for energy storage capacitors ${ }^{1}$, packaging, adhesive and coatings in electrical sensors, ion-exchangers ${ }^{2-4,8-13}$, semiconductors ${ }^{5}$, activators, catalyst and thermally stable materials ${ }^{6,7,26}$.

Extensive research work has been carried out on the synthesis, characterization and thermogravimetry of terpolymers. Jadhav have synthesized and studied the thermal degradation properties of terpolymers based on 2,2'-biphenol ${ }^{14,15}$. Pratik Michael et al. studied the structural and thermal degradation of terpolymer derived from salicylic acid, guanidine and 
formaldehyde ${ }^{16}$. Kobayashi and Kon-ishi synthesized resorcinol-acetone copolymer and studied its thermogravimetric analysis ${ }^{17}$. Singru and coworkers reported thermoanalytical study and kinetics of 8-hydroxyquinoline-5-sulphonic acid-oxamide-formaldehyde and $p$-cresol-oxamideformaldehyde terpolymer resins ${ }^{18,19}$. Patel and coworkers synthesized and characterized acrylic copolymers derived from 8-quinolinyl methacrylate and studied its ion exchange properties ${ }^{20}$. Peterson et al. have carried out thermogravimetric analysis in inert as well as oxidative atmosphere $^{21}$. The thermal stability of polymers and copolymers has been extensively studied by employing the method of thermogravimetric analysis by several authors ${ }^{22-25}$.

Study of thermal behaviour of terpolymers in air atmosphere at different temperature provides useful information about the nature of species produced at various temperatures due to degradation. Non-isothermal thermogravimetric analysis in which sample is subjected to condition of continuous increase in temperature at linear rate which provides the useful information about the degradation pattern and thermal stability.

Literature studies have revealed that no terpolymer has been synthesized using the monomer 2,2'-biphenol, tetraethylenepentamine and formaldehyde. In the present article, we present the synthesis of phenolic straight chain and branched terpolymers (2,2'-BP-TEPAF) by condensation terpolymerization reaction and characterized by elemental analysis, UV-visible, FT-IR, ${ }^{1} \mathrm{H}$ NMR spectra. Studies were further extended to thermogravimetric analysis in order to predict its thermal degradation pattern and kinetic parameters by implementing Friedman, Chang and Sharp-Wentworth method.

\section{Experimental}

2,2'-Biphenol and tetraethylenepentamine are of analytical grade purity which was purchased from Acros Chemicals, Belgium. Formaldehyde (37\%) was purchased from SD Fine Chemicals, India. All the used solvents like $N, N$-dimethylformamide, dimethyl sulphoxide, tetrahydrofuran, acetone, diethyl ether were procured from Merck, India.

\section{Synthesis}

2,2'-BPTEPAF terpolymer resins were prepared by condensing 2,2'-biphenol and tetraethylenepentamine with formaldehyde in presence of $2 \mathrm{M} \mathrm{NaOH}$ as a catalyst in the various molar proportions of reacting monomers at $145 \pm 1{ }^{\circ} \mathrm{C}$ in an oil bath for six hrs of continuous heating. The temperature of electrically heated oil bath was controlled with the help of a dimmerstat. A wine red coloured solution was obtained after completion of reaction and the yellowish resinous solid product was formed after re-precipitation with $2 \mathrm{M} \mathrm{HCl}$. The product was immediately removed, filtered and repeatedly washed with cold-distilled water, dried in air and powdered with the help of an agate mortar and pestle. The product obtained was extracted with diethyl ether to remove excess of 2,2'-biphenol-formaldehyde copolymer which might be present along with 2,2'-BPTEPAF terpolymer resin. Dried resin sample was dissolved in $8 \% \mathrm{NaOH}$ and regenerated using 1:1 $\mathrm{HCl} /$ water $(\mathrm{v} / \mathrm{v})$ with constant stirring then it was filtered. This process was repeated twice. Resulting terpolymer resin sample was washed with boiling water and dried in a vacuum at room temperature. Purified terpolymer resin was finely ground to pass through 300 -mesh size sieve and kept in a vacuum over silica gel $^{26}$.

Similarly, other terpolymer resins viz. 2,2'-BPTEPAF-II, 2,2'-BPTEPAF-III were synthesized by varying the molar proportion of starting material, i.e. 2,2-biphenol, tetrethylenepentamine with formaldehyde in the ratio of $1: 2: 4$ and $4: 1: 5$. The chemical reaction of 2,2'-BPTEPAF-I terpolymer resin is given in Figure 1. Synthesis and physical data of 2,2'-BPTEPAF terpolymer resins are shown in Table 1. 


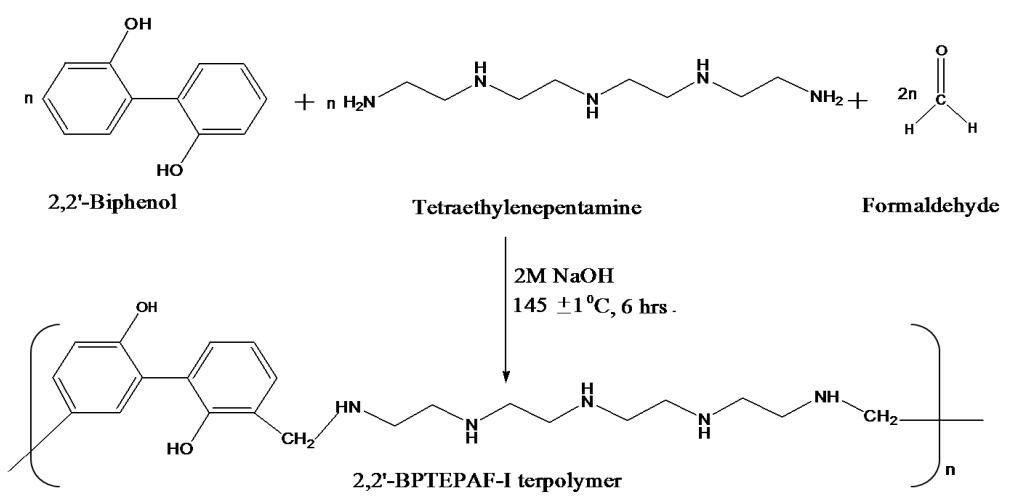

Figure 1. Chemical reaction of 2,2'-BPTEPAF- I terpolymer resin

Table 1. Synthesis and physical data of 2,2'-BPTEPAF terpolymer resins

\begin{tabular}{cccccc}
\hline \multirow{2}{*}{$\begin{array}{c}\text { Terpolymer } \\
\text { resins }\end{array}$} & $\begin{array}{c}\text { 2,2'-Biphenol, } \\
\text { mol. }\end{array}$ & $\begin{array}{c}\text { Tetraethylene- } \\
\text { pentamine, mol. }\end{array}$ & $\begin{array}{c}\text { Formal- } \\
\text { dehyde,mol. }\end{array}$ & $\begin{array}{c}\text { Catalyst } \\
\text { 2 M NaOH } \\
\text { (aq) } \mathrm{mL}\end{array}$ & $\begin{array}{c}\text { Yield, } \\
\%\end{array}$ \\
\hline 2,2'-BPTEPAF-I & 0.1 & 0.1 & 0.2 & 200 & 79.00 \\
2,2'-BPTEPAF-II & 0.1 & 0.2 & 0.4 & 200 & 81.05 \\
2,2'-BPTEPAF-III & 0.4 & 0.1 & 0.5 & 200 & 83.65 \\
\hline
\end{tabular}

\section{Spectral and thermal studies}

Terpolymer resins were subject to elemental analysis for carbon, hydrogen and nitrogen on Elmentar Vario EL III Elemental Analyzer at Sophisticated Test and Instrumentation Centre, Cochin University of Science and Technology, Cochin. UV-Vis spectra of terpolymer resins in DMSO solvent recorded on JASCO V-630 UV-VIS spectrophotometer at Nagpur in the range of 200-850 $\mathrm{nm}$ at Department of Pharmacy, R.T.M. Nagpur University, Nagpur. ${ }^{1} \mathrm{H}$ NMR studies were performed in DMSO as solvent on Bruker Advance-II 400 NMR spectrophotometer and FT-IR spectra were recorded in nujol mull on Perkin Elmer spectrum RX-I spectrophotometer in the range of $4000-500 \mathrm{~cm}^{-1}$ at Sophisticated Analytical Instrumentation Facility (SAIF) Punjab University, Chandigarh. The non-isothermal thermogravimetric analysis of newly prepared terpolymer resins has been carried out using Perkin Elmer Diamond 3 II thermogravimetric analyzer in air atmosphere with a heating rate at $10{ }^{\circ} \mathrm{C} \mathrm{min}-1$ in the temperature ranges from $40-1000{ }^{\circ} \mathrm{C}$ at VNIT, Nagpur.

\section{Thermal analysis}

Dynamic (non-isothermal) thermogravimetric analysis of all terpolymer resins have been carried out in air atmosphere with heating rate of $10{ }^{\circ} \mathrm{C} \mathrm{min}^{-1}$ by taking $5-6 \mathrm{mg}$ of samples in platinum crucible. Thermograms were recorded in the temperature range $40-1000{ }^{\circ} \mathrm{C}$. Thermal activation energy (Ea), order of reaction (n) and frequency factor $(\mathrm{z})$ has been calculated from thermogravimetric data.

\section{Theoretical considerations}

Thermogram expresses the dependence of change in mass on the temperature which gives information about sample composition, product formed after heating and kinetic parameters. Kinetics parameters have been determined using Friedman ${ }^{27}$, Chang ${ }^{28}$ and Sharp-Wentworth ${ }^{29}$ techniques as follows: 


\section{Friedman technique}

$$
\ln \left(\frac{d \alpha}{d t}\right)=\ln (z)+n \ln (1-\alpha)-\frac{E \alpha}{R T}
$$

Where, $\alpha$ is the conversion at time $t$; $R$ is the gas constant $(8.314 \mathrm{~J} / \mathrm{mol} / \mathrm{K})$ and $\mathrm{T}$ is the absolute temperature (K). From the slope of the linear plot of $\ln (1-\alpha) v s .1 / T, n$ can be obtained. The plot of $\ln (\mathrm{d} \alpha / \mathrm{dt}) v s .1 / \mathrm{T}$ should be linear with the slope $\mathrm{E}_{\mathrm{a}} / \mathrm{R}$, from which $\mathrm{E}_{\mathrm{a}}$ can be obtained.

\section{Chang technique}

$$
\frac{\ln \left(\frac{d \alpha}{d t}\right)}{(1-\alpha)^{n}}=\ln (z)-\frac{E \alpha}{R T}
$$

A plot of $\left[\ln (\mathrm{d} \alpha / \mathrm{dt}) /(1-\alpha)^{\mathrm{n}}\right]$ vs. $1 / \mathrm{T}$ will yield a straight line if the order of decomposition reaction, $\mathrm{n}$ is selected correctly. The slope and intercept of this line will provide the $\left(-E_{a} / R\right)$ and $\ln (z)$ values, respectively.

Sharp-Wentworth technique

$$
\frac{\log \left(\frac{d c}{d t}\right)}{1-c}=\log \left(\frac{A}{\beta}\right)-\frac{E \alpha}{2.303 R} \frac{.1}{T}
$$

Where, $\mathrm{dc} / \mathrm{dt}=$ rate of change of fraction of weight with change in temperature; $\beta=$ linear heating rate i.e. $\mathrm{dT} / \mathrm{dt} ; \mathrm{c}=$ fraction of polymer decomposed at time $\mathrm{t}$.

Thus, a linear plot of $\frac{\log \left(\frac{d c}{d t}\right)}{1-c}$ versus $\frac{1}{T}$ is obtained whose slope gives the value of $\mathrm{E}_{\mathrm{a}}$ and A may be evaluated from the intercept. The linear relationship confirmed that the assumed order is correct.

\section{Results and Discussion}

\section{Elemental analysis}

The composition of terpolymer resins was assigned on the basis of elemental analysis and was found to be in good agreement with that of calculated values as shown in Table 2.

Table 2. Elemental analysis data of 2,2'-BPTEPAF terpolymer resins

\begin{tabular}{cccccccccc}
\hline \multirow{2}{*}{ Terpolymer resins } & \multirow{2}{*}{$\begin{array}{c}\text { Monomer } \\
\text { empirical }\end{array}$} & Monomer & \multicolumn{6}{c}{ Elemental analysis, \% } \\
\cline { 5 - 9 } & empirical & \multicolumn{3}{c}{$\mathrm{C}$} & \multicolumn{4}{c}{$\mathrm{H}$} & \multicolumn{3}{c}{$\mathrm{N}$} \\
\cline { 5 - 9 } & formula & Weight & Cal. & Exp. & Cal. & Exp. & Cal. & Exp. \\
\hline 2,2'-BPTEPAF-I & $\mathrm{C}_{22} \mathrm{H}_{37} \mathrm{~N}_{5} \mathrm{O}_{4}$ & 435 & 60.68 & 60.99 & 8.5 & 8.35 & 16.09 & 16.21 \\
2,2'- BPTEPAF-II & $\mathrm{C}_{32} \mathrm{H}_{62} \mathrm{~N}_{10} \mathrm{O}_{5}$ & 666 & 57.65 & 57.86 & 9.30 & 9.36 & 21.02 & 21.16 \\
2,2'- BPTEPAF-III & $\mathrm{C}_{61} \mathrm{H}_{64} \mathrm{~N}_{5} \mathrm{O}_{10}$ & 1026 & 71.34 & 71.45 & 6.23 & 6.42 & 6.82 & 7.67 \\
\hline
\end{tabular}

\section{UV-Visible spectra}

UV-visible spectra of the 2,2'-BPTEPAF terpolymer resins give rise to a similar pattern is represented in Figure 2. The spectra of these terpolymer resins exhibit two absorption maxima at 270 to $280 \mathrm{~nm}$ and 310 to $330 \mathrm{~nm}$. These observed positions for the absorption bands have different intensities. The more intense band which may be accounted for $\pi \rightarrow \pi^{*}$ allowed transition of conjugation in aromatic ring. While the later and less intense band due to $\mathrm{n} \rightarrow \pi^{*}$ forbidden transitions of $-\mathrm{OH}$ group. 
The auxochromic substituents $(-\mathrm{OH})$ interacting with $\pi$ electron of the benzene ring. This interaction stabilizes $\pi^{*}$ state and thus lowers the energy as a result bathochromic shift is caused. The presence of phenolic hydroxyl group is responsible for bathochromic shift (shift toward longer wavelengths). Both bathochromic and hyperchromic effect of conjugation shown by chromophore (aromatic ring) and auxochrome $(-\mathrm{OH})$ group in repeated unit of terpolymer resins.

\section{FT-IR spectra}

The FT-IR spectra of 2,2'-BPTEPAF terpolymer resins are depicted in Figure 3 and IR spectral data are tabulated in Table 3. Broad and strong band appeared at 3351-3397 $\mathrm{cm}^{-1}$, which may be assigned to the stretching vibration of the phenolic - $\mathrm{OH}$ groups exhibiting intermolecular hydrogen bonding ${ }^{30,31}$. Short and weak band at $3055-3062 \mathrm{~cm}^{-1}$ are indicate the presence of a $>\mathrm{NH}$ stretching (sec. amine) group. A medium broad band, displayed at $1578-1602 \mathrm{~cm}^{-1}$, may be due to bending vibration of secondary amine. Substituted aromatic ring show medium and short bands at $1482-1484 \mathrm{~cm}^{-1}$. Medium and short peak displayed at $1371-1374 \mathrm{~cm}^{-1}$ suggested the presence of $-\mathrm{CH}_{2}$ - bridge in terpolymer resins ${ }^{32}$. The presence of $\mathrm{C}-\mathrm{N}$ stretch can be accounted by the presence of band at $1154-1155 \mathrm{~cm}^{-1}$ and $1143-1047 \mathrm{~cm}^{-1}$. The presence of substitution on aromatic ring also shows sharp peak at $756-758 \mathrm{~cm}^{-1}$.

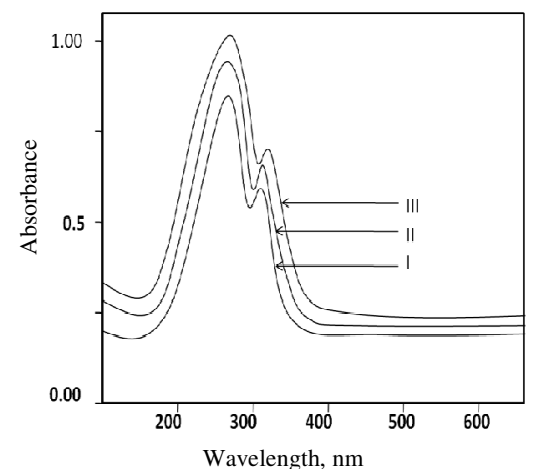

Figure 2. UV-Visible spectra of terpolymer resins 2,2'-BPTEPAF

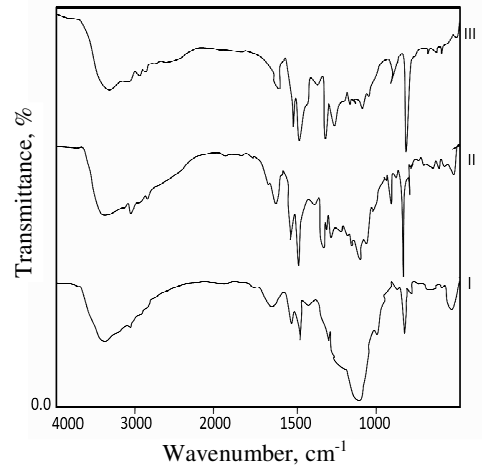

Figure 3. IR spectra of terpolymer resins 2,2'-BPTEPAF

Table 3. IR frequencies spectral data of 2,2'-BPTEPAF terpolymer resins

\begin{tabular}{|c|c|c|c|}
\hline Assignment & 2,2'-BPTEPAF-I & $\begin{array}{l}\text { IR Wavenumber, } \mathrm{cm}^{-1} \\
\text { 2,2'-BPTEPAF-II }\end{array}$ & 2,2'-BPTEPAF-III \\
\hline -OH (Phenolic) & 3397 b,st & $3355 \mathrm{~b}, \mathrm{st}$ & $3361 \mathrm{~b}, \mathrm{st}$ \\
\hline $\begin{array}{l}\text { >NH stretching } \\
\quad \text { (sec.amine) }\end{array}$ & 3062 sh,w & $3056 \mathrm{sh}, \mathrm{w}$ & $3055 \mathrm{sh}, \mathrm{w}$ \\
\hline $\begin{array}{c}\text { Secondary } \\
\text { amine bending }\end{array}$ & $1602 \mathrm{~m}, \mathrm{~b}$ & $1587 \mathrm{~m}, \mathrm{~b}$ & $1578 \mathrm{~m}, \mathrm{~b}$ \\
\hline$-\mathrm{CH}_{2}$ - bridge & $1371 \mathrm{~m}, \mathrm{sh}$ & $1372 \mathrm{~m}, \mathrm{sh}$ & $1374 \mathrm{~m}, \mathrm{sh}$ \\
\hline C-N stretch & $\left.\begin{array}{c}1155.57 \mathrm{~m} \\
1047.81 \mathrm{st}, \mathrm{b}\end{array}\right\}$ & $\left.\begin{array}{l}1154 \mathrm{~m} \\
1043 \mathrm{sh}\end{array}\right\}$ & $\left.\begin{array}{l}1155 \mathrm{~m} \\
1043 \mathrm{~m}\end{array}\right\}$ \\
\hline $\begin{array}{c}\text { Substituted } \\
\text { aromatic ring }\end{array}$ & $758 \mathrm{sh}$ & $756 \mathrm{sh}$ & $755 \mathrm{sh}$ \\
\hline
\end{tabular}




\section{${ }^{1}$ H NMR spectra}

${ }^{1} \mathrm{H}$ NMR spectral data of terpolymer resins are incorporated in Table 4 and spectrum is presented in Figure 4. Spectra reveal different patterns of peaks, since each of them possesses a set of protons having different proton environment. A singlet is observed due to proton of phenolic $\mathrm{OH}$ group, observed at $\delta 7.99-8.19 \mathrm{ppm}$. A singlet is observed at $\delta 7.31-7.36 \mathrm{ppm}$ and is due to unsymmetrical pattern of protons of phenol. A signal appeared in the region 2.51-2.56 ppm may be due to proton of methelenic bridges $\left(\mathrm{Ar}-\mathrm{CH}_{2}-\mathrm{N}\right)$ of polymer chain shows singtet. The singlet in the region 6.80-6.88 ppm is attributed to proton of - $\mathrm{NH}$ - bridge.

Table 4. ${ }^{1} \mathrm{H}$ NMR spectral data of 2,2'-BPTEPAF terpolymer resins

\begin{tabular}{lccc}
\hline \multicolumn{1}{c}{$\begin{array}{c}\text { Nature of protons } \\
\text { assigned }\end{array}$} & \multicolumn{2}{c}{${ }^{1} \mathrm{H}$ NMR Chemical shift $(\delta)$ ppm of Terpolymer resins } \\
& 2,2'-BPTEPAF-I & 2,2'-BPTEPAF-II & 2,2'-BPTEPAF-III \\
\hline $\begin{array}{l}\text { Proton of Ar-OH } \\
\text { (phenolic-OH) }\end{array}$ & $7.99 \mathrm{~s}$ & $8.1 \mathrm{~s}$ & $8.19 \mathrm{~s}$ \\
$\begin{array}{l}\text { Aromatic proton } \\
\text { (unsymm. pattern) }\end{array}$ & $7.36 \mathrm{~s}$ & $7.34 \mathrm{~s}$ & $7.31 \mathrm{~s}$ \\
$\begin{array}{l}\text { Methylene proton of d, } \\
\text { Ar-CH }{ }_{2}-\mathrm{NH} \text { moiety }\end{array}$ & $2.56 \mathrm{~s}$ & $2.54 \mathrm{~s}$ & $2.51 \mathrm{~s}$ \\
Proton of -NH bridge & $6.88 \mathrm{~s}$ & $6.83 \mathrm{~s}$ & $6.80 \mathrm{~s}$ \\
\hline
\end{tabular}

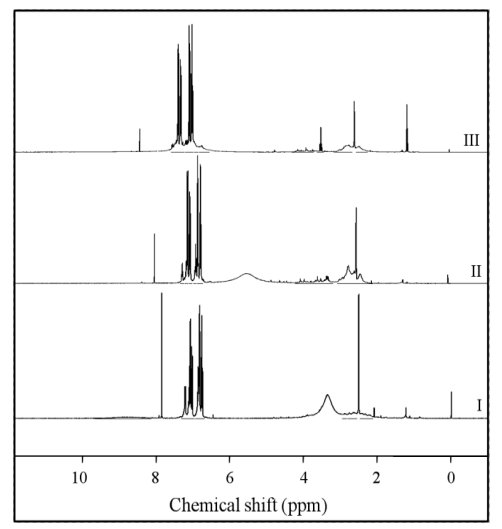

Figure 4. ${ }^{1} \mathrm{H}-\mathrm{NMR}$ spectra of terpolymer resins 2,2 '-BPTEPAF

\section{Thermogravimetric analysis}

Decomposition pattern of terpolymer resin is shown in Figure 6. Thermogram of 2,2'BPTEPAF-I terpolymer exhibited four step decomposition in the temperature range $40-1000{ }^{\circ} \mathrm{C}$. The first step is of slow decomposition between 40 to $140{ }^{\circ} \mathrm{C}$ corresponds to $8.15 \%$ loss which may be attributed to loss of two water molecules which may be due to moisture entrapped in the terpolymer resin ${ }^{33,34}$ against calculated $8.27 \%$ present per repeat unit of polymer. The second step decomposition starts from $140{ }^{\circ} \mathrm{C}$ to $220{ }^{\circ} \mathrm{C}$ which represents degradation of two hydroxyl groups (16.21\% found and $16.09 \%$ calculated). The third step of decomposition starts from 220 $310{ }^{\circ} \mathrm{C}$ corresponds to $57.28 \%$ loss of biphenyl ring against calculated $57.01 \%$. Fourth step starts from 310 to $1000{ }^{\circ} \mathrm{C}$ corresponding to loss of complete tetraethylenepentamine moiety. Table 6 represents the thermal degradation data for 2,2'-BPTEPAF-I, II, III terpolymer resins. To obtain the kinetic parameters, the thermal degradation kinetic equations described by Sharp-Wetworth, Friedman and Chang was adopted. By using thermal decomposition data 
and then applying above methods the activation energy (Ea), order of reaction (n) and Frequency factor $(\mathrm{z})$ are calculated. The activation energies calculated by these methods are depicted in Table 5. The thermal stability of terpolymer predicted on the basis of the initial decomposition temperature is in concurrence with that predicted from the activation energy values. From the above spectral studies, the proposed structures of 2,2'-BPTEPAF-I, II, III terpolymer resins are given below.

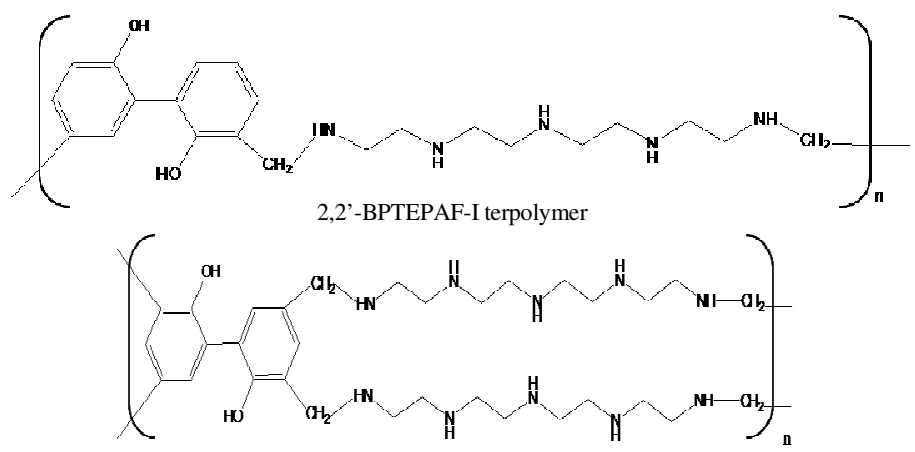

2,2'-BPTEPAF-II terpolymer

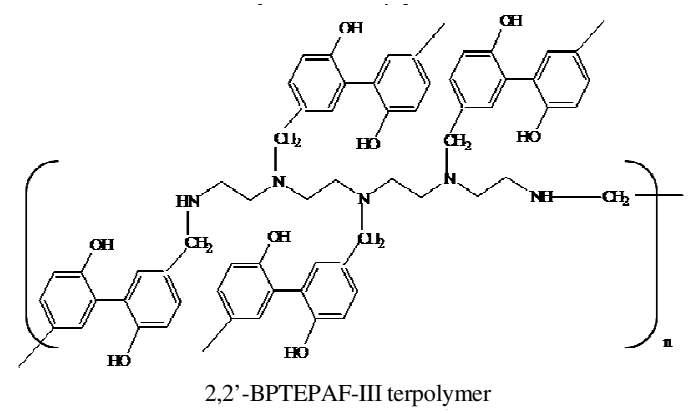

Figure 5. Structures of 2,2'-BPTEPAF-I, II, III terpolymer resins

Table 5. Thermoanalytical data for each degradation step of 2,2'-BPTEPAF- I terpolymer resin

\begin{tabular}{cccccccccc}
\hline \multirow{2}{*}{ Kinetic equations } & \multicolumn{10}{c}{ Decomposition steps } \\
\cline { 2 - 10 } & \multicolumn{2}{c}{ 2,2-BPTEPAF-I } & \multicolumn{2}{c}{ 2,2-BPTEPAF-II } & \multicolumn{2}{c}{ 2,2-BPTEPAF-III } \\
\cline { 2 - 10 } & $\mathrm{E}_{\mathrm{a}}$ & $\mathrm{n}$ & $\mathrm{z}$ & $\mathrm{E}_{\mathrm{a}}$ & $\mathrm{N}$ & $\mathrm{z}$ & $\mathrm{E}_{\mathrm{a}}$ & $\mathrm{n}$ & $\mathrm{z}$ \\
\hline Friedman & 10.81 & 1.8 & 11.09 & 10.90 & 1.4 & 10.91 & 12.97 & 1.7 & 11.29 \\
Chang & 11.11 & 1 & 11.14 & 11.40 & 1 & 10.98 & 13.63 & 1 & 11.29 \\
Sharp-Wentworth & 46.79 & 1 & 4.81 & 47.16 & 1 & 4.73 & 56.13 & 1 & 4.91 \\
\hline
\end{tabular}

Kinetics of thermal decomposition by Friedman, Chang and Sharp-Wentworth techniques

The analysis of thermograms indicates that the decomposition of terpolymer is four step process in which loss of water molecules present per repeat unit of polymer in first step. Due to fragmentation of straight chain and branch linked structure of terpolymer resins because of which it doesn't takes more time to attain the thermal equilibrium as well as at $1000{ }^{\circ} \mathrm{C}$ degradation process occurs up to final level without leaving behind the remaining moiety. The thermal stability of 2,2'-BPTEPAF terpolymer resins are concluded to be higher, may be due to the stronger intermolecular hydrogen bonding present in polymer structures because of 
moisture entrapped in the terpolymer resin which would be more difficult to break and hence more resistant to higher temperature or it may be due to possibility of an linear and branched structure of terpolymer chain which gives stability to polymer chain.

Kinetic parameters for 2,2'-BPTEPAF terpolymer resins have been calculated using Friedman, Chang and Sharp-Wentworth method. In Friedman method Activation energy $\left(E_{a}\right)$ of three terpolymer resins have been obtained from the plot between $\ln (\mathrm{d} \alpha / \mathrm{dt}) v s$. $(1 / \mathrm{T})$ and order of reaction (n) from the plot between $\ln (1-\alpha) v s$. (1/T) as shown in Figures 7 and 8 respectively. Values of $\ln (\mathrm{z})$ are calculated at each temperature region with the help of Ea and $\mathrm{n}$.

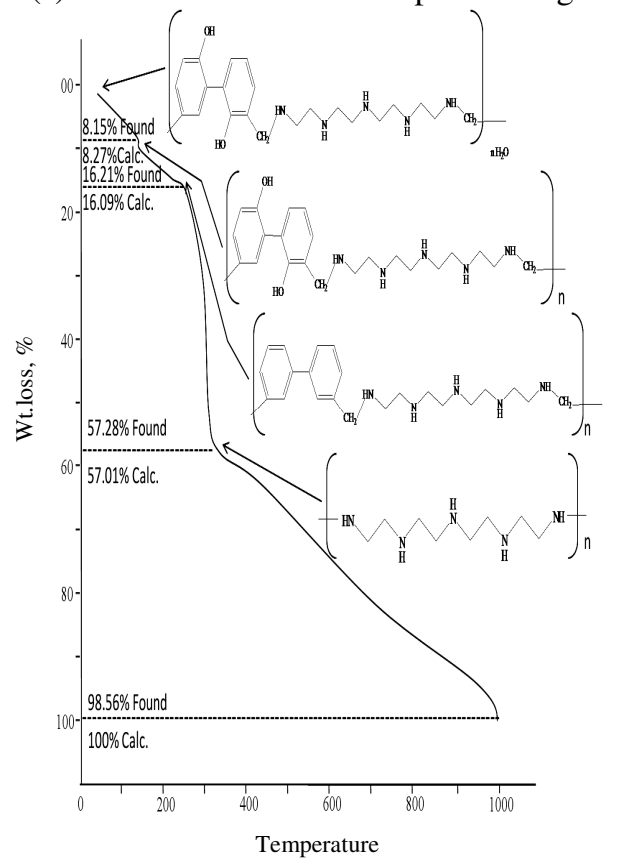

Figure 6. Decomposition pattern of 2,2'-BPTEPAF- I terpolymer resin

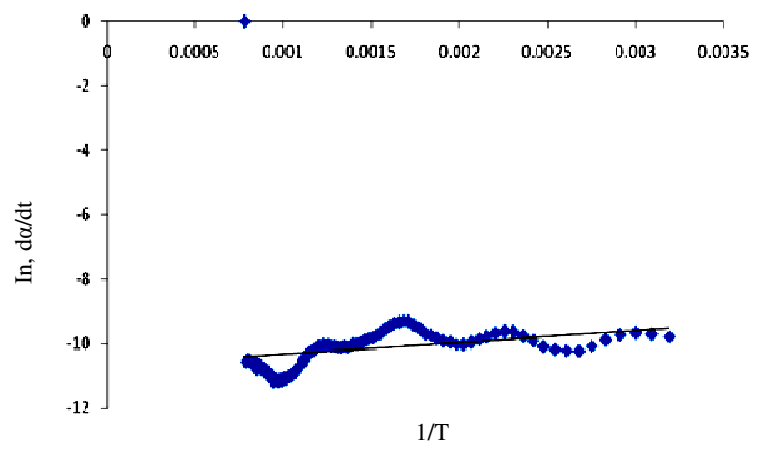

Figure 7. Friedman activation energy plot of 2,2'-BPTEPAF- I terpolymer resin

Figure 9 shows Chang method gives plots between $\left[\ln (\mathrm{d} \alpha / \mathrm{dt}) /(1-\alpha)^{\mathrm{n}}\right] v s$. $(1 / \mathrm{T})$ for 2,2'-BPTEPAF at the heating rate $10{ }^{\circ} \mathrm{C} \cdot \mathrm{min}^{-1}$ which is used to calculate $\mathrm{E}_{\mathrm{a}}$ and $\ln (\mathrm{z})$ of respective degradation reaction for best fitted value of $\mathrm{n}$ (from Friedman equation), which corresponds to correct reaction order for each respective thermal decomposition step. 


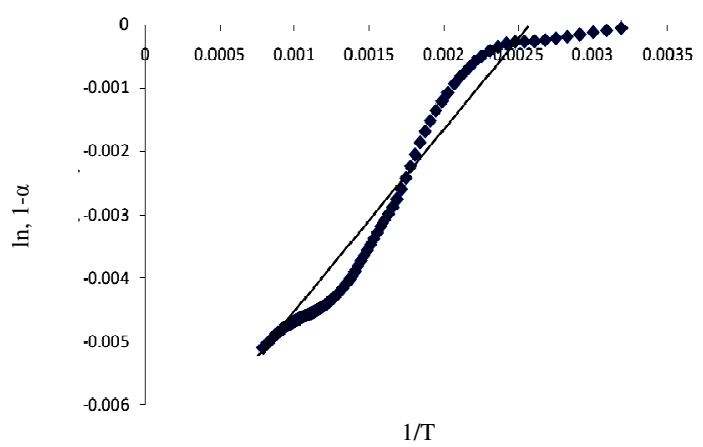

Figure 8. Friedman order of reaction plot of 2,2'-BPTEPAF- I terpolymer resin

Also kinetic parameters for different thermal degradation zones have been calculated by Sharp-Wentworth method. $\mathrm{E}_{\mathrm{a}}$ and $\ln (\mathrm{z})$ values are calculated from the plot between $\frac{\log \left(\frac{d c}{d t}\right)}{1-c}$ $v s . \frac{1}{T}$ with best fitted values of $\mathrm{n}$ for each respective degradation $\mathrm{n}$ reaction (Figure 10).

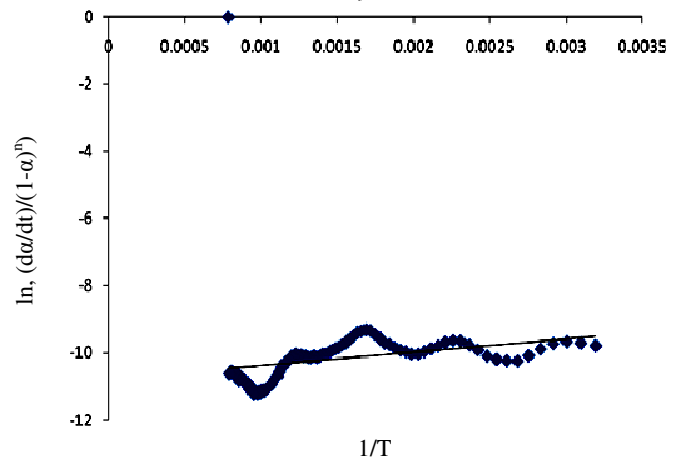

Figure 9. Chang plot of 2,2'-BPTEPAF- I terpolymer resin

By using thermal decomposition data and then applying the Friedman method (representative Friedman plots of 2,2'-BPTEPAF terpolymer resin are shown in Figures 7 and 8. Activation energy is calculated which is in agreement with the activation energy is calculated by Chang plot (Figure 9) for the polymer has been shown.

A plot of percentage mass loss $v s$. temperature is shown in Figure 5 for a representative 2,2'-BPTEPAF terpolymer resin. From the TG curves, the thermoanalytical data and decomposition temperature has been determined for different stages as given in Table 6.

This kinetic analysis should be a starting point to obtain the useful information on the behavior of samples. Fairly comparable results in the kinetic parameters i.e. $\mathrm{E}_{\mathrm{a}}$ and $\mathrm{z}$ are obtained by Friedman and Chang may be due to analogy in mathematical model but somewhat different observations are observed in Sharp-Wentworth method.

The energy of activation calculated by Fridman and Chang method are found to be in will agreement with each other. But Shrp-Wentworth method shows different observations. Order of reaction calculated from Sharp-Wentworth and Chang method is found to be one. But order of reaction calculated by Friedman method not obeys first order kinetics perfectly as reported by Jacobs and Tompkin ${ }^{35}$ and Coats and Redfern ${ }^{36}$. 
Table 6. Thermal degradation behavior of 2,2'-BPTEPAF-I terpolymer resin

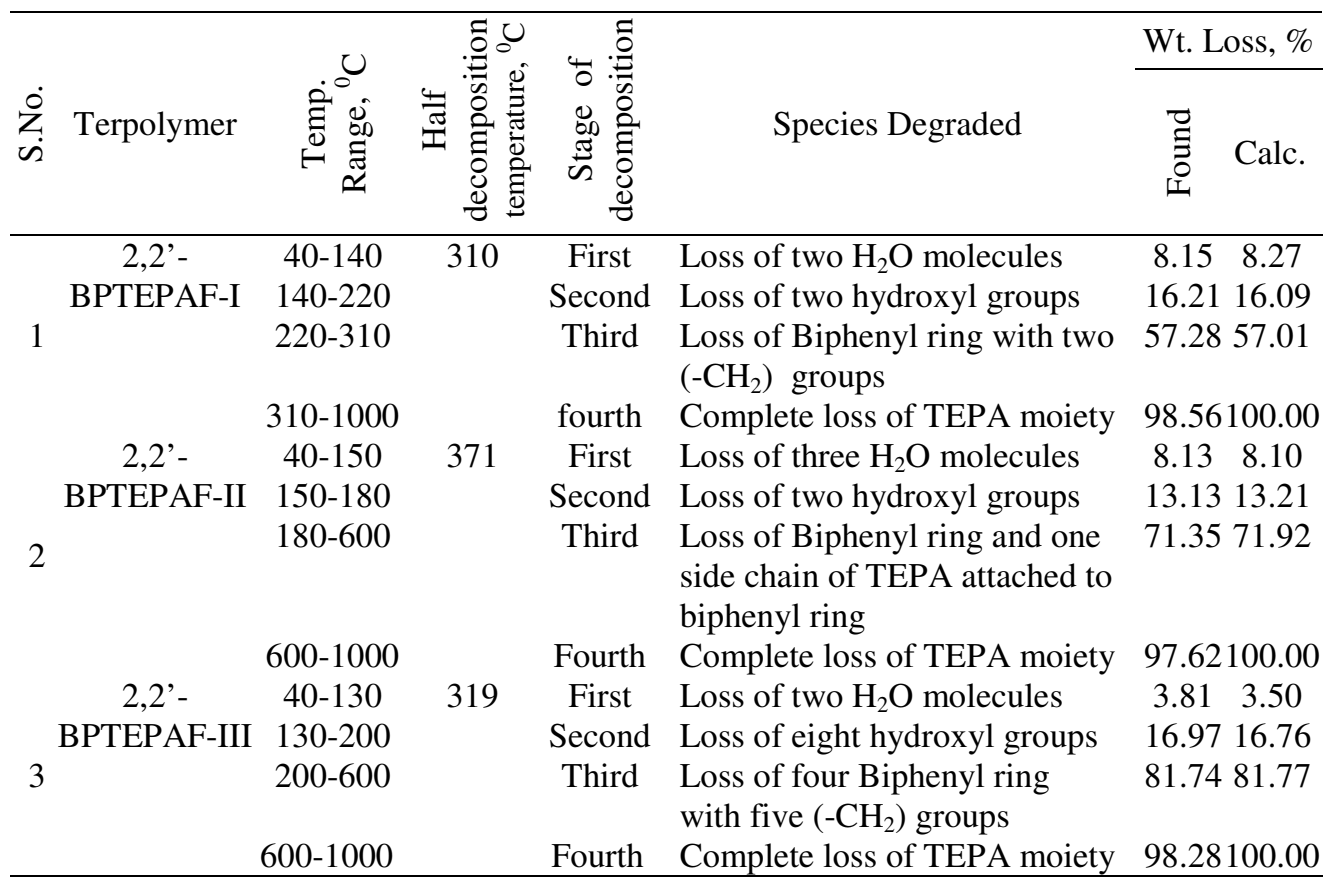

The thermogravimetric analysis of all the three terpolymer resins prepared has been carried out but, the thermal data and kinetic plots for only one representative case are given in Figures 7-10.

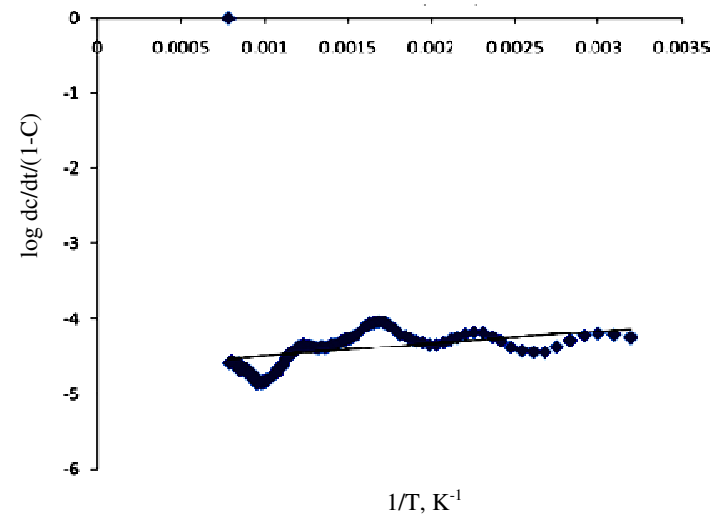

Figure 10. Sharp-Wentworth plot of 2,2'-BPTEPAF -I terpolymer resin

By using above mentioned techniques fairly good straight-line plots are obtained which represents versatility and great utility of thermal degradation mathematical equations in thermal studies and attempts are developing to implement the model free kinetic equations.

\section{Conclusion}

Synthesis of targeted terpolymer resins (2,2-BPTEPAF) has been confirmed which is supported by the results obtained by the elemental analysis and spectral data. Presence of 
spectral peaks of methylene bridge in the spectral data confirms the formation of terpolymer resins. The thermogram shows four degradation steps for all the terpolymers i.e. in first step loss of water molecule, in second step hydroxyl group, in third step biphenyl ring and in fourth step loss of complete tetraethylenepentamine moiety. The activation energy values calculated from Friedman and Chang method are in well agreement with each other due to resemblance in the mathematical model and with some variation in Sharp-Wentworth method.

\section{Acknowledgement}

The authors wish to express their sincere thanks to University Grants Commission (UGC) for providing the financial assistance to our laboratory. The authors also thankful to Dr. S. D. Dawande, Director in-Charge, Laxminarayan Institute of Technology, Nagpur for providing necessary laboratory facility.

\section{References}

1. Wesley Hackenberger, Ed., Alberta, Paul W Rehrig, Daeyong Jeong and Qiming Zhang CARTS: USA, Palm Springs, CA, 2005, 21-24.

2. Gurnule W B, Rahangdale P K, Paliwal L J and Kharat R B, J Appl Polym Sci., Wiley Periodicals, Inc. 2003, 89, 787-790.

3. Mane V D, Wahane N J and Gurnule W B, J Appl Polym Sci., Wiley Periodicals, Inc. 2009, 111(6), 3039-3049.

4. Tarase M V, Zade A B and Gurnule W B, J Appl Polym Sci., Wiley Periodicals, Inc. 2008, 108(2), 738-746.

5. Kushwaha A D, Hiwase V V and Kalambe A B, Der Pharma Chemica, 2012, 4(1), 557-567.

6. Kapse S K, Hiwase V V and Kalambe A B, Der Pharma Chemica, 2012, 4(1), 460-467.

7. Conley R T, Am Chem Soc Div Org Plat Chem Pap., 1966, 26, 138.

8. Raval D K, Narola B N and Patel A J, Iran Polym J., 2005, 14(9), 775-784.

9. Jin-Yin W and Wan-yuan C, Huaxue Yu Nianhe, 2002, 1, 20-21.

10. Egorov A N, Sukhorukov Y I, Plotnikova G V and Khaliullin A K, Rus J Appl Chem., 2002, 75, 152.

11. Claus M, Eur Pat EP 1, 2001, 254, 937.

12. Beauvais R A, Alexandratos S D, React Funct Polym., 1998, 36(2), 113-123.

13. Patel S A, Shah B S, Patel R M and Patel P M, Iran Polym J., 2004, 13(6), 445-453.

14. Jadhao M M, Paliwal L J and Bhave N S, J Appl Polym Sci., Wiley Periodicals, Inc. 2006, 101(1), 227-232.

15. Jadhao M M, Paliwal L J and Bhave N S, J ApplPolym Sci., Wiley Periodicals, Inc. 2005, 96(5), 1605-1610.

16. Pratik E P Michael, Lingala Peter S, Juneja H D and Paliwal L J, J Appl Polym Sci., Wiley Periodicals, Inc. 2004, 92, 2278-2283.

17. Ataru Kobayashi and Gen-ichi Konishi, Molecules, 2009, 14(1), 364-377.

18. Singru R N, Khati V A, Gurnule W B, Zade A B and Dontulwar J R, Anal Bioanal Electrochem., 2011, 3(1), 67-86.

19. Singru R N, Scholars Research Library Der Pharma Chemica, 2011, 3(5), 128-134.

20. Patel S A, Shah B S, Patel R M and Patel P M, Iran Polym J., 2004, 13(6), 445-453.

21. Peterson J D, Vyazovkin S and Wight C A, Macro Mol Chem Phys., 2001, 202, 775.

22. Urade D N, Hiwase V V and Kalambe A B, J Chem Pharm Res., 2012, 4(1), 732-740.

23. Singru R N, International Scholarly Research Network ISRN Thermodynamics, 2012, Article ID 323916, 8; DOI:10.5402/2012/323916 
24. Khedkar K M, Hiwase V V, Kalambe A B and Deosarkar S D, J Chem., 2012, 9(4), 1911-1918.

25. Tarase M V, Zade A B and Gurnule W B, J Appl Polym Sci., Wiley Periodicals, Inc. 2010, 116(2), 619-627.

26. Manavalan R and Patel M M, Die Makromolekulare Chemie, 2003, 184(4), 717-723.

27. Freidman H L, J Polymer Sci., 1964, C6, 183.

28. Chang W L, J Appl Polym Sci., 1994, 53(13), 1759-1769.

29. Sharp J B and Wentworth S A, Anal Chem., 1969, 41(14), 2060-2062.

30. Nakanishi K, Infra Red Absorption Spectroscopy Practical; Nolden Day: INC and Nankodo Co. Ltd. Tokyo, 1967.

31. Vogel A I, Text book of practical organic chemistry; Longman Scientific and Technical: UK, 1989.

32. Stuart B H, Infrared Spectroscopy; Fundamentals and applications: Wiley, U. K., 2004.

33. Patel M M and Manavalan R, Indian J Chem Soc., 1983, 22, 117-119.

34. Patel M M and Manavalan R, Indian J Chem Soc., 1984, 61, 490-494.

35. Jacobs P W M and Tompkin F C, Chemistry of solid state; WI Graver: London, 1995, 188.

36. Coats A and Redfern J P, J Polym Sci Part B: Polym Phys., 1965, 3(3), 917-932. 